\title{
CLINICO-DEMOGRAPHIC PATTERNS OF DEPRESSION AND ANXIETY IN CHILDREN AND ADOLESCENTS
}

\author{
Anamaria Ciubara, Roxana Chirita, Stefan Lucian Burlea, Ancuta Ignat, \\ Smaranda Diaconescu, Ilinca Untu, Valeriu V. Lupu \\ "Gr. T. Popa" University of Medicine and Pharmacy, Iasi
}

\begin{abstract}
In both adults and pediatric population, depression is a major disability cause; suicide is the third death cause among children and adolescents. Whereas depression and depression with associated anxiety are among the most common psychiatric pathologies in children and adolescents (with strong impact on their global functionality and with a significant influence on their development process), they are far from studied thoroughly. This study aims to highlight certain demographic and clinical patterns associated to depression of anxiety in children and adolescents, which may represent premises for broader future research and for the consolidation of primary, secondary, and tertiary prevention methods.
\end{abstract}

Keywords: depression, anxiety, children and adolescents, suicide

\section{INTRODUCTION}

WHO's "Health for the world's adolescents" report reveals that depression is the predominant cause of illness and disability for both boys and girls aged 10 to 19 years old. Depression is the number one cause of illness in this category (10$19)$, while suicide is the third mortality cause. According to WHO, should adolescents receive proper treatment in time, it could prevent death and spare them from pain that lasts for the rest of their lives $(1,2)$.

Though depression among children and adolescents is one of the most common mental health disorders, it has not been paid due attention. Scientific research on pediatric population is scarce, while prevention programs have not been developed sufficiently. According to a study conducted in Romania by the "Save the Children" Organization, launched in 2010, 20\% of children suffer from a psychiatric condition, $3.5 \%$ display clinical depression, while $13 \%$ report anxiety disorders. In Roma- nia, there are 880,709 children with psychiatric disorder, of whom 154,124 with depression and 572,461 with anxiety disorders (1).

\section{THEORETICAL BACKGROUND}

Depressive disorder among children and adolescents is a real public health issue, with increasing prevalence and strong impact upon the global functionality and psychological, cognitive, and social development of the individual. Depressive disorders have a $2-3 \%$ occurrence among children and of $8 \%$ among adolescents worldwide, hence the fundamental need for early diagnostic and intervention. Whereas major depression has an important genetic component, complex studies have reported that $40-50 \%$ of the cases are due to exogenous stressors and to negative events in the life of children and adolescents. Despite the fact that pediatric depression symptoms are similar to those of adults, the clinical manifestations of the first are closely connected to patients' development level $(1,2,3)$. 
According to DSM-5, the same diagnostic criteria are considered as for adults; however, in case of children and adolescents, depressive mood may be replaced by irritability. As in case of adults, the main risk entailed by depressive spectrum disorders is suicidal behaviour; the incidence increases by age (for pediatric population) $(2,4)$. One or more of the following symptoms are signs of depression if they are persistent (most of the day for at least two weeks), thus influencing the social implication of the child and determining various degrees of discomfort: irritability, anger bursts, manifest behaviour disorders, social withdrawal, lack or increase of appetite, sadness and helplessness, insomnias or hypersomnias, hypoprosexia, crying easily, somatic complaints, reduction of global functionality, suicidal behaviour/suicidal ideation (5-7).

Concerning anxiety spectrum disorders, they are under-diagnosed in all development stages. Often, children with pathological anxiety may actually display no psychiatric symptoms, but they may be concealed by somatic signs, such as headaches, gastrointestinal discomfort, palpitations, dryness of mucous, etc. $(2,8)$. A major problem of anxiety in children is precisely its persistence over time, with significant impact upon patients' global functionality. The most important comorbidity of anxiety in children and adolescents is precisely depression (the coexistence of the two psychiatric conditions was reported in approximately $30 \%$ of the cases) $(1,9)$.

To already known etiologic factors (depicted in previous studies), new factors were added in the context of social, economic, and even political changes of the last 20 years. These new underlying or favouring factors have major implication in the physical and mental development of adolescents (they may determine from adaptive deficit to suicide). Acknowledging them and then introducing effective - primary, secondary, and tertiary - intervention programs represent essential elements in the strategy of medical psychiatric and pediatric healthcare for this age category.

\section{OBJECTIVES OF THE STUDY}

This study aims to assess the demographic structure of patients aged between 12 and 18, with secondary diagnostic of mental disorder set in "St. Mary" Children Emergency Hospital Iaşi. We focused on patients with various forms of depression, manifested for three years (2,088 records). Another goal was to pinpoint the interrelation between depression level and anxiety in adolescents, by focusing of background and gender differences.
Considering the severe under-diagnostic of depression among pediatric population, this study attempts to identify clinical, causal, and demographic patterns meant to help detect this psychiatric disorder early and design efficient prevention systems.

\section{MATERIAL AND METHODS}

In order to attain the proposed goals, we conducted a retrospective-descriptive study to assess the demographic variables of patients with secondary psychiatric diagnostics within "St. Mary" Children Hospital and with primary psychiatric diagnostics admitted to the Pediatric Neuropsychiatry Ward of the "Socola" Hospital. The assessment methods for subjects included in the research were observation, documentation, and questionnaire, as well as specific depression of anxiety assessment scales (HAMD; HAMA).

The statistical processing of data was conducted using SPSS 15.0. Research hypotheses concern the existence of a significant positive correlation between depression and anxiety; they also postulate the higher incidence of anxiety and depression pathology among females of the pediatric population, as well as background differences. Therefore, the independent variables of the study comprise gender (female/male), background (urban/rural), while dependent variables concern the level of anxiety and depression.

Inclusion criteria were age between 12-18 for patients admitted in the period 2011-2014 in various clinics of "St. Mary" Children Hospital and in the Pediatric Neuropsychiatry Ward of the "Socola" Hospital.

\section{FINDINGS AND DISCUSSIONS}

This study includes 483 cases of mixed anxietydepressive disorder, which represent secondary diagnostics, because patients were admitted to "St. Mary" Children Hospital Iasi for various somatic symptoms. The Neuropsychiatry Ward of the "Socola" Hospital Iasi recorded 71 cases of pediatric patients diagnosed with mixed anxiety-depressive disorder.

Upon analyzing the patient series of the two hospitals, females recorded slightly more cases of mixed anxiety-depressive disorder (this ratio is also present among adults). However it must be mentioned that among patients admitted to the Neuropsychiatry Ward of the "Socola" Hospital Iasi, the males actually recorded slightly higher incidence. 
This can be explained by the higher aggressive and negative behavioural potential of boys, exacerbated by specific irritable mood of anxiety spectrum disorders among children and adolescents. $\mathrm{T}$ test for independent samples recorded the following results: $\mathrm{t}(118)=2.12, \mathrm{p}<0.05$, and this analysis enabled the identification of significant differences between depression in females and in males among pediatric population (Fig. 1, Fig. 2).

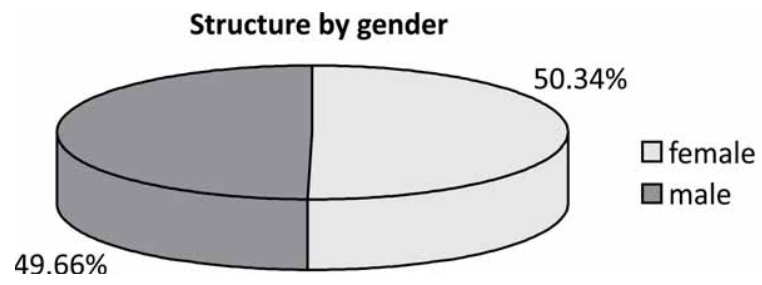

FIGURE 1. Distribution by gender of mixed anxietydepressive disorders among children and teenagers

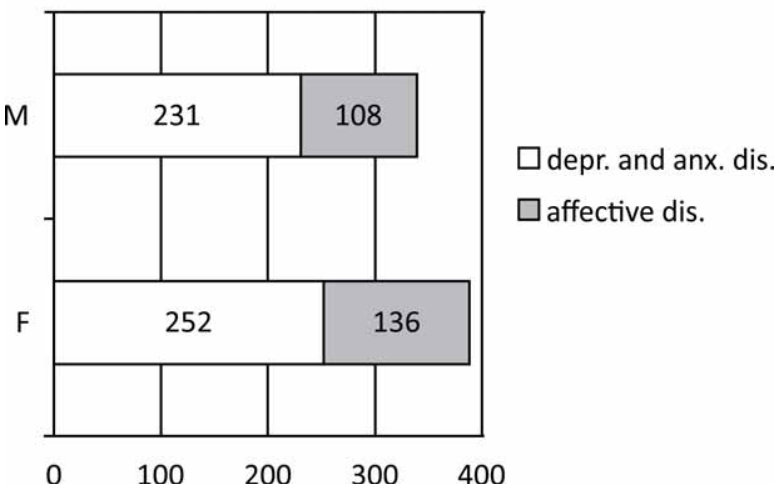

FIGURE 2. Number of diagnosed cases by gender

The comparative analysis of statistical data concerning the age variable indicates that in "St Mary" Children Hospital Iasi most cases of mixed anxiety-depressive disorder is recorded at the age of 12 (19\% of the patients), while in the Neuropsychiatry Ward of the "Socola" Hospital Iasi, the ages of 13, 16 , and 17 represent each $18 \%$ of all patients recorded in this ward. In the pediatric hospital, the age with the lowest number of depression cases is 18 , which coincides with the age of passage toward adulthood, reason for which most patients are redirected to specialized clinics (Fig. 3, Fig. 4).

Upon analysing the statistical data, a slight dominance of urban patients was found within the study series taken into account in this research. However, these figures may actually reflect that rural children do not have proper access to medical services in general and to psychiatric services in particular. Hence, the under-diagnostic degree of mixed anxiety-depressive disorder is significant. In addition, purely human values and support strategies of rural communities may be more effective

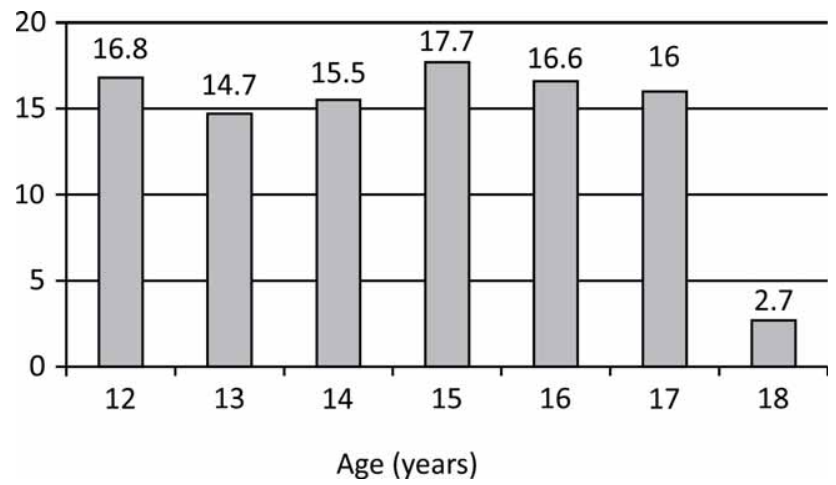

FIGURE 3. Distribution of cases by age

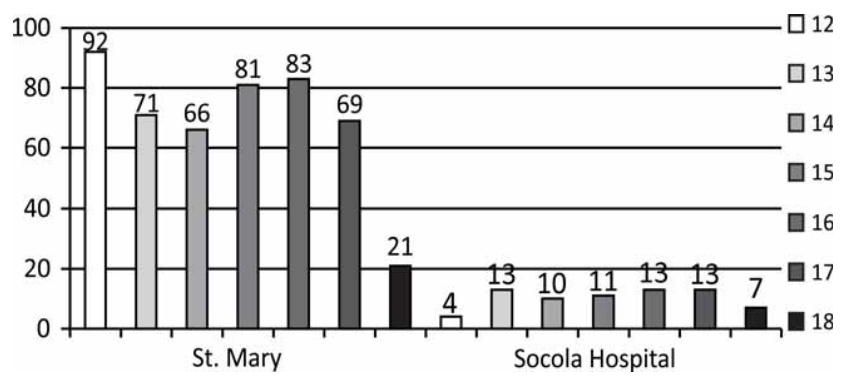

FIGURE 4. Mixed anxiety-depressive disorder diagnostics by age

in terms of psychological help; the belonging to this group may be assimilated as a protective resource that could be highlighted within prophylactic programs for depression of anxiety among pediatric population. The system of social relationships and the increasing academic demands may turn the urban environment into a vulnerability factor for depression and anxiety among children and adolescents. In order to validate this hypothesis, the T test for independent samples was applied: $\mathrm{t}(118)=0.88$ $p>0.05$. Hence, whereas the comparison between the means of the two samples shows a difference between depression levels among urban adolescents compared to rural adolescents, a significant difference between them cannot be ascertained.

In order to set the depression diagnostics, we used the nosological categories of mixed anxietydepressive disorder and of reactive depression syndrome. The reactive depression syndrome diagnostic corresponded to $85.92 \%$ of the cases. This illustrated the dominance of psycho-traumatizing events as determinants of depressive symptomatology. Mixed anxiety-depressive disorder was present in $14.08 \%$ of all cases within the study (Fig. 5).

Concerning the cases admitted to the paedo-psychiatric ward, $40.89 \%$ were accompanied by selfharming attempts upon admission: this is a major psychiatric emergency and it represents the deepest expression of moral pain and lack of hope associated to depression. Moreover, it is worth underscor- 


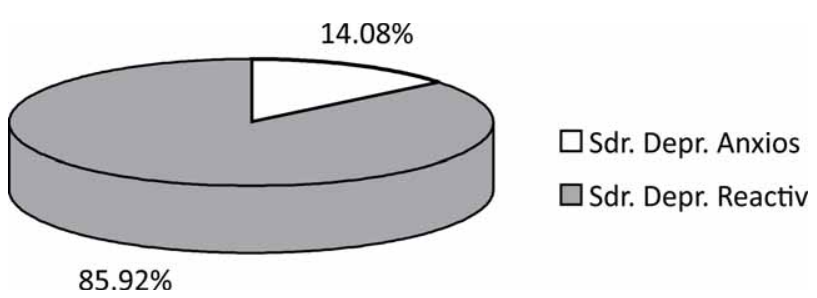

FIGURE 5. Types of depressive disorder

ing that, upon analyzing the medical history of these patients, it was found that $61.5 \%$ displayed (at least once in their life) suicidal behaviour (Fig. 6).

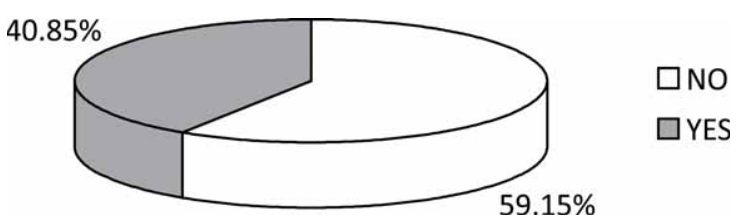

FIGURE 6. Suicide attempts

Aspects such as displaying depressive symptoms and seeking socio-psychological support are different in the age group 12-15 compared to 1618. Most patients of the first group agreed to consult a psychologist or a psychiatrist, compared to patients of the second group, who proved very reluctant to such ideas (this can be explained by the specifics of social relationships established in this period of life, based on the approval and validation of the dominant group). This aspect represents a fundamental impediment in the early diagnostic of depression in adolescents.

Furthermore, to test the hypothesis concerning the relationship between depression and anxiety among children and adolescents, Pearson's correlation was used ( 0.84 was obtained, which denotes a variability in the same sense of both depression and anxiety). Thus, a significant positive correlation was found between the two variables: $p$ is lower than 0.05 . Consequently, statistical analysis shows that the possibility of associating anxiety increases proportionally with scores indicating average-severe depression. The correlation Graph is an ascending cloud of points from bottom-left to topright. This aspect confirms that the relation between the anxiety variable and the depression variable is positive. Had there not existed such a relation, the points would have been distributed evenly on the Graph; similarly, had the relation been inversely proportional, the cloud would have been descending (Fig. 7).

\section{CONCLUSIONS}

The data obtained are heterogeneous, which is due to changes in the diagnostic criteria, to the scarce number of studies conducted on the pediatric population in the field of psychiatric conditions (depression and anxiety, implicitly), to the "cohort" phenomenon, and to differences related to the size of study series and to the instruments used.

An important percentage of adolescents with depression or depression with anxiety complications are under-diagnosed, which shows the need to implement depression screening program for adolescents within pediatric/paedo-psychiatric services or family medicine services.

The close and positive correlation between depression and anxiety is useful for designing diagnostic and therapy plans, and mostly for elaborating psycho-prophylactic plans. Chronic or acute stress caused by traumatising life experiences may generate distress, which can be manifested by depression associated to other disorders: anxiety, aggressiveness, and panic attacks (the association between depression and anxiety was proved by this study). At the same time, sociodemographic factors influence the onset and evolution of this nosological category; consequently, they can be used to implement a depression screening for adolescents.

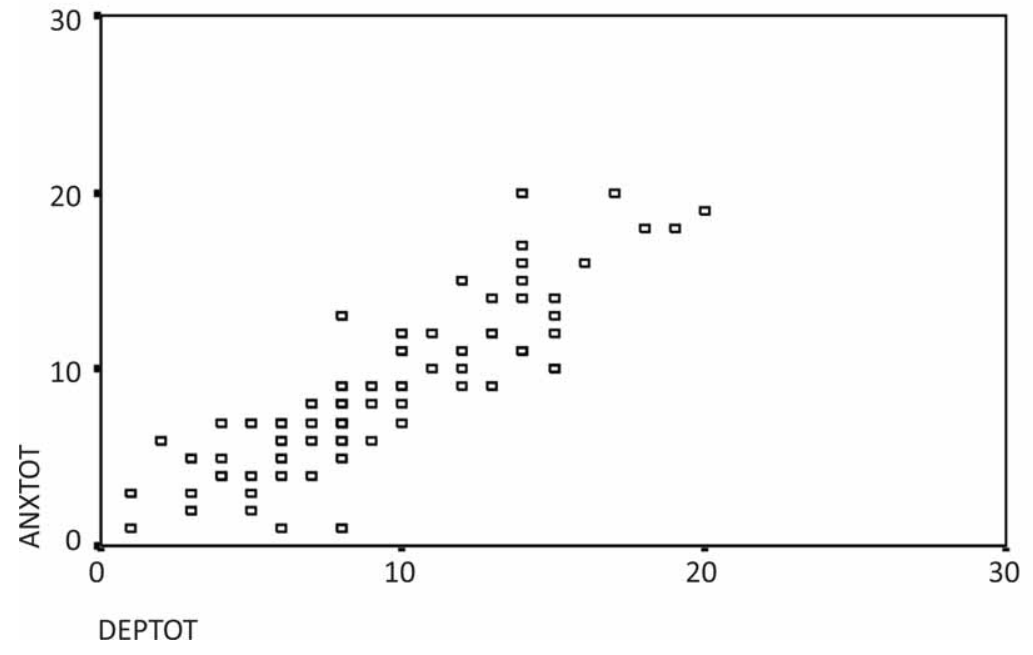

FIGURE 7. Relation between depression and anxiety in children and adolescents 
This study also demonstrates that adolescents have a hard time accepting to consult a psychiatrist, in the context of relational clichés based on which they function.

A warning signal is precisely the high incidence of self-harming behaviour among pediatric population, which is closely connected to depression, as well as the evidence of reactive depression as dominant condition. This indicates, implicitly, the possibility of prevention and the need to implement early detection means for this pathology among young children and adolescents.

The clinico-demographic patterns associated to anxiety-depressive disorders may represent a premise for future studies aiming at getting better insight into depression and anxiety predictors among pediatric population. The final goal is to implement effective primary, secondary, or tertiary prevention means.

\section{REFERENCES}

1. http://www.who.int/

2. American Psychiatric Association (2013). Diagnostic and statistical manual of mental disorders (DSM-5) Washington, D.C.: American Psychiatric Pub; 2013

3. Starr L.R., Conway C.C., Hammen C.L., Brennan P.A. Transdiagnostic and disorder-specific models of intergenerational transmission of internalizing pathology. Psychol Med. 2014; 44(01):161-172

4. Saluja G., lachan R., Scheidt P.C., Overpeck M.D., Sun W., Giedd J.N. Prevalence of and risk factors for depressive symptoms among young adolescents. Arch Pediatrics Adolesc Med. 2004; 158(8):760-765

5. Hollingworth S.A., Burgess P.M., Whiteford H.A. Affective and anxiety disorders: prevalence, treatment and antidepressant medication use. Aust NZJ Psychiatry. 2010; 44(6):513-519

6. Cuijpers P., Smits N., Donker T., ten Have M., de Graaf R. Screening for mood and anxiety disorders with the five-item, the three-item, and the two-item Mental Health Inventory. Psychiatry Res. 2009; 168(3):250-255

7. Kaltiala-Heino R., Frojd S. Correlation between bullying and clinical depression in adolescent patients. Adolescent health, medicine and therapeutics. 2011; 2:37-44

8. Luukkonen A.H., Rasanen P., Hakko H., Riala K. Bullying behavior in relation to psychiatric disorders and physical health among adolescents: a clinical cohort of 508 underage adolescents in Northern Finland. Psychiatry Res. 2010; 178:166-170(a)

9. Seals D., Young J. Bullying and victimization: prevalence and relationship to gender, grade level, ethnicity, self-esteem, and depression. Adolescence. 2003; 38:735-747 